\title{
CAMBIOS RECIENTES EN LA ESTRUCTURA TERRITORIAL SOCIODEMOGRÁFICA DEL ÁREA METROPOLITANA DE VALENCIA (2001-2011) ${ }^{1}$
}

\author{
Julia Salom \\ Félix Fajardo \\ Instituto Interuniversitario de Desarrollo Local \\ Departamento de Geografía \\ Universidad de Valencia \\ Julia.Salom@uv.es, Felix.Fajardo@uv.es
}

\section{RESUMEN}

Desde principios de siglo, las áreas metropolitanas españolas se han visto afectadas por intensos procesos socioeconómicos y territoriales, que han tenido un fuerte impacto en su estructura interna. El análisis de la evolución de las desigualdades sociodemográficas internas en el Área Metropolitana de Valencia durante el periodo intercensal 2001-2011 indica que, como consecuencia de estos procesos, se ha producido un cambio en el número y significado de los componentes principales explicativos de la segregación espacial en el área, así como el incremento de los contrastes sociodemográficos internos, y un aumento de la complejidad del modelo territorial.

Palabras clave: Estructura interna metropolitana, Segregación urbana, Área Metropolitana de Valencia.

Fecha de recepción: febrero 2015.

Fecha de aceptación: septiembre 2015.

1 El presente artículo se ha realizado en el marco del proyecto "Sostenibilidad y Competitividad Urbanas en un contexto global. El Área Metropolitana de Valencia” (CSO2013-46863-C3-1-R), financiado por la Secretaría de Estado de Investigación, Desarrollo e Innovación del Ministerio de Economía y Competitividad, dentro del Programa Estatal de Investigación, Desarrollo e Innovación Orientada a los Retos de la Sociedad. Uno de los autores, Félix Fajardo, cuenta con una ayuda para contratos predoctorales para la formación de profesorado universitario, convocatoria 2014 (FPU14/05550), financiada por el Ministerio de Educación, Cultura y Deporte. 


\section{ABSTRACT}

Since the beginning of the century, the Spanish metropolitan areas have been affected by intense territorial and socio-economic processes, which have had a strong impact on its internal structure. An analysis of the evolution of the internal socio-demographic disparities in the Metropolitan Area of Valencia during the inter-census period 2001-2011 indicates that, as a result of these processes, there has been a change in the number and significance of the main explanatory components of spatial segregation in the area, as well as the increase in the internal demographic contrasts and in the complexity of the territorial model.

Keywords: Metropolitan internal structure, Urban Segregation, Metropolitan Area of Valencia.

\section{INTRODUCCIÓN: DESIGUALDAD Y SEGREGACIÓN ESPACIAL EN LOS ESPACIOS URBANOS}

El estudio de las desigualdades sociales internas en las áreas metropolitanas y su plasmación espacial es un tema recurrente en los estudios de geografía urbana. Partiendo de los trabajos iniciales que pretendían modelizar la estructura interna de los espacios urbanos en base a criterios ecológicos (Burguess, 1929; Hoyt, 1939), y de los enfoques a menudo más descriptivos que explicativos de la ecología factorial de las áreas urbanas (Slevky y Bell, 1955), las perspectivas de análisis se han enriquecido y hecho más complejas con las reflexiones propiciadas por los efectos de la globalización y el postfordismo sobre la desigualdad social intrametropolitana.

Los cambios económicos recientes han generado un aumento de la polarización social en las ciudades que ha sido especialmente intenso en aquéllas que han experimentado de forma más intensa los procesos de terciarización (Harvey, 1990). Por otra parte, el aumento de las migraciones internacionales ha dado lugar a nuevos problemas y tensiones sociales asociadas a la diversidad étnica y cultural en ciudades, como las españolas, que hasta el momento no las habían experimentado de forma acusada. Esta polarización social ha tenido una expresión territorial, dando lugar a lo que se ha denominado como "ciudad dual" o "ciudad dividida" (Sassen, 1991; Molenkopf y Castells, 1991), un entorno en donde han proliferado también expresiones territoriales de autosegregación como las comunidades valladas y los desarrollos residenciales privados (Bellet, 2007). Por otro lado, a las tradicionales dimensiones de diferenciación residencial (estatus socioeconómico, estatus familiar y estatus étnico), se han añadido nuevos ejes de formación de desigualdad (sexo, edad, sexualidad cultural, identidad cultural, etc.) que convierten la estructura social metropolitana en un puzzle complejo al que Soja ha denominado "ciudad fractal" (Soja, 2008, p. 384-386).

La segregación urbana entendida como la fragmentación del espacio urbano en células diferenciadas, incluso jerarquizadas, de grupos sociales homogéneos (Ocaña, 2005) tiene como corolario negativo la formación de barrios vulnerables, definidos como espacios afectados por "la combinación de múltiples dimensiones de desventaja, en el que toda esperanza de movilidad social ascendente, de superación de su condición social de exclusión o próxima a ella, es contemplada como extremadamente difícil de alcanzar" (Hernández Aja, 2007, p. 8). Estas desventajas se articulan en torno a cuatro ejes básico: el demográfico (envejecimiento y presencia de 
hogares unipersonales y monoparentales), el económico (desempleo, precariedad laboral, bajo nivel de formación), el residencial (barrios degradados e infraviviendas) y el subjetivo (sensación de inseguridad, insuficiencia de zonas verdes, ruido, etc.) (Instituto Juan de Herrera, 2010).

En el caso de España, la crisis económica ha supuesto una vuelta de tuerca adicional en las tendencias citadas, ya que el deterioro del mercado de trabajo y los recortes en los servicios sociales han afectado gravemente a las clases trabajadoras y medias, generando un grupo creciente de "nuevos pobres". Como consecuencia de este proceso, la desigualdad no ha dejado de aumentar en España desde el inicio en 2007 de la crisis inmobiliaria y financiera (FOESSA, 2013). Las cotas históricas de desempleo, la pérdida de poder adquisitivo de los hogares españoles y en general el debilitamiento del Estado del Bienestar como consecuencia de las políticas de austeridad, han aumentado la vulnerabilidad de la sociedad española. Esta vulnerabilidad ha tenido una traslación espacial en el interior de las ciudades, dando lugar a "geografías locales de la crisis" que profundizan en la fragmentación social previamente existente entre distintos sectores de la ciudad (Méndez y Prada, 2014).

En respuesta a estos cambios, se han realizado en los últimos años numerosos estudios que han analizado fundamentalmente los efectos de la inmigración en la segregación urbana en distintas ciudades españolas (Del Pino, 2001; Bayona, 2007; Echazarra, 2008; Goñi, 2008; Martori y Hoberg, 2008; Natera, 2010; Díaz, 2010; Domínguez et al., 2010), aunque también existen algunos otros centrados en la segregación de las clases con mayor poder adquisitivo y la formación de las denominadas "ciudadelas" (Rubiales, Bayona y Pujadas, 2012), con un especial énfasis en los desarrollos residenciales privados y las denominadas “comunidades valladas" (Bellet, 2007).

Los métodos de análisis aplicados han ido desde el cálculo de distintos índices de segregación, en particular de los propuestos en el trabajo clásico de Massey y Denton (1988) y el estudio de los factores geográficos asociados a dicha segregación (Bayona, 2007; Echazarra, 2008; Natera, 2010; Domínguez et al., 2010), hasta la aplicación de nuevas técnicas de estadística espacial para detectar los agrupamientos (Martori y Hoberg, 2008; Díaz, 2010), pasando por los más clásicos análisis de definición de tipologías y agrupamiento de unidades territoriales de características socioeconómicas similares, normalmente mediante técnicas cluster o de análisis discriminante, precedidos o no por un análisis factorial o de componentes principales que permite sintetizar la masa de datos inicial en unas cuantas variables o componentes significativos (Del Pino, 2001; Goñi, 2008).

Aunque algunos de estos trabajos, sobre todo los centrados en las pautas residenciales de la población inmigrante, han adoptado una perspectiva dinámica, no existen estudios que permitan comparar los efectos de los cambios sociales y económicos más recientes sobre la conformación de la estructura sociodemográfica interna de las áreas metropolitanas, probablemente debido a la escasez de los datos disponibles. El Censo de 2011, pese a sus limitaciones técnicas, nos ha permitido abordar este tema desde una perspectiva comparativa en relación con la situación existente en 2001, fecha del último censo anterior. La citada fuente nos permite analizar, convenientemente complementada con información procedente del Padrón de Habitantes de las mismas fechas, los cambios experimentados por la estructura sociodemográfica de los espacios residenciales urbanos tras un periodo compuesto por una fase de fuerte crecimiento económico y demográfico (2001-2007) seguido por una etapa de crisis económica y social (2008-2011). 
En el presente artículo estudiamos los cambios experimentados en un área metropolitana española de tamaño medio, la de Valencia, partiendo de la hipótesis de que los procesos recientes antes descritos han tenido consecuencias en la estructura interna del área metropolitana desde el punto de vista de las características sociodemográficas de la población residente.

\section{FUENTES Y METODOLOGÍA}

El objetivo del presente trabajo es el estudio de la estructura interna actual del Área Metropolitana de Valencia (AMV) desde el punto de vista de las características de la población residente y de su evolución reciente desde el año 2001 mediante el análisis de los componentes y variables socioeconómicas y demográficas más relevantes. Para ello, se ha realizado un análisis de componentes principales y un posterior agrupamiento cluster a partir de la información referida a variables definitorias de aspectos socioeconómicos, demográficos y residenciales de los municipios del AMV para dos fechas clave: 2001 y 2011, con el fin de comparar los resultados obtenidos y analizar los cambios acaecidos en dicho periodo.

La información utilizada procede de los Censos de Población y Viviendas de los años 2001 y 2011 y de los Padrones de Población de los años 2002 y 2012. El recurso a ambas fuentes de forma complementaria permite obtener información a escala más detallada sobre los aspectos demográficos y de lugar de nacimiento de la población residente. La información se ha obtenido a partir de la Base de Datos "en línea” del Instituto Nacional de Estadística (www.ine.es).

El cambio metodológico en la elaboración de los Censos de los años 2001 (censo clásico) y 2011 (censo muestral) ha exigido realizar un laborioso proceso de selección y homogeneización de la información disponible a fin de construir una base de datos con información comparable entre ambas fechas. Cabe añadir la limitación impuesta en el Censo del año 2011 a la hora de analizar unidades espaciales con volúmenes de población absoluta reducidos, debido a elevados errores de muestreo derivados del procedimiento de recogida de datos. Esto ha supuesto una importante restricción en cuanto a la selección de la información de partida, ya que algunas de las variables susceptibles de ser incluidas en el análisis han tenido que ser descartadas al no estar disponibles en el Censo del año 2011 a escala municipal o inferior. Los ámbitos temáticos más afectados por estas limitaciones han sido la estructura socioprofesional, la procedencia por nacionalidades de la población y los equipamientos de la vivienda. La selección final (ver tabla 1) incluye 26 variables indicativas de:

- Estructura y situación socioeconómica de la población (ocupación por sectores, tasa de paro)

- Nivel de estudios de la población

- Estructura de edades y tamaño del hogar

- Nacionalidad de la población residente

- Características de la vivienda (régimen de tenencia, superficie, antigüedad, equipamiento).

El ámbito territorial de estudio es el Área Metropolitana de Valencia delimitada por José $\mathrm{M}^{\mathrm{a}}$ Feria (Feria, 2008) desde una concepción funcional del espacio urbano, en la que la varia- 
ble de movilidad residencia-trabajo es el elemento clave para la delimitación de los espacios metropolitanos. De la delimitación original se han excluido aquellos municipios que, debido a su reducido tamaño demográfico, no presentaban un número mínimo de variables en el año 2011 que permitiera la obtención de resultados significativos en el análisis estadístico realizado ${ }^{2}$.

Tabla 1

CONJUNTO DE VARIABLES SELECCIONADAS PARA EL ANÁLISIS FACTORIAL

\begin{tabular}{|c|c|c|}
\hline \multicolumn{2}{|c|}{ INDICADORES } & VARIABLES \\
\hline \multirow{2}{*}{$\begin{array}{l}\text { Estructura de } \\
\text { edades y tamaño } \\
\text { del hogar }\end{array}$} & $\begin{array}{l}\text { Estructura de } \\
\text { edades }\end{array}$ & $\begin{array}{l}\text { Porcentaje de población de } 0 \text { a } 14 \text { años } \\
\text { Porcentaje de población de } 15-29 \text { años } \\
\text { Porcentaje de población de } 65 \text { o más años }\end{array}$ \\
\hline & Tamaño del hogar & $\begin{array}{l}\text { Porcentaje de hogares con } 1 \text { miembro, } \\
\text { Porcentaje de hogares con } 5 \text { o más miembros } \\
\text { Tamaño medio del hogar }\end{array}$ \\
\hline Nivel de estudios & $\begin{array}{l}\text { Nivel de estudios } \\
\text { población mayor } \\
\text { de } 16 \text { años }\end{array}$ & $\begin{array}{l}\text { Porcentaje de población analfabeta } \\
\text { Porcentaje de población sin estudios } \\
\text { Porcentaje de población con estudios de primer grado } \\
\text { Porcentaje de población con estudios de tercer grado }\end{array}$ \\
\hline \multirow{2}{*}{$\begin{array}{l}\text { Estructura } \\
\text { y situación } \\
\text { socioeconómica }\end{array}$} & $\begin{array}{l}\text { Relación } \\
\text { preferente con la } \\
\text { actividad }\end{array}$ & Tasa de desempleo \\
\hline & Rama de actividad & $\begin{array}{l}\text { Porcentaje de población ocupada en agricultura ganadería y pesca } \\
\text { Porcentaje de población ocupada en industria } \\
\text { Porcentaje de población ocupada en servicios }\end{array}$ \\
\hline Nacionalidad & $\begin{array}{l}\text { Grupos de } \\
\text { nacionalidad }\end{array}$ & $\begin{array}{l}\text { Porcentaje de extranjeros sobre el total de la población } \\
\text { Porcentaje de nacionales de la Unión Europea sobre el total de los extranjeros, } \\
\text { Porcentaje de nacionales de países africanos sobre el total de los extranjeros, } \\
\text { Porcentaje de nacionales de países americanos sobre el total de los extranjeros }\end{array}$ \\
\hline \multirow{4}{*}{$\begin{array}{l}\text { Características de } \\
\text { la vivienda }\end{array}$} & $\begin{array}{l}\text { Régimen de } \\
\text { tenencia }\end{array}$ & $\begin{array}{l}\text { Porcentaje de viviendas con hipoteca } \\
\text { Porcentaje de viviendas en alquiler }\end{array}$ \\
\hline & Equipamiento & Porcentaje de viviendas sin calefacción \\
\hline & $\begin{array}{l}\text { Año de } \\
\text { construcción }\end{array}$ & $\begin{array}{l}\text { Porcentaje de viviendas construidas entre 1900-1960 } \\
\text { Porcentaje de viviendas construidas entre 1961-2001 } \\
\text { Edad media de la vivienda }\end{array}$ \\
\hline & Superficie útil & $\begin{array}{l}\text { Porcentaje de viviendas de menos de } 90 \mathrm{~m}^{2} \\
\text { Porcentaje de viviendas de más de } 120 \mathrm{~m}^{2}\end{array}$ \\
\hline
\end{tabular}

2 Estos municipios han sido Domeño, Olocau, Petrés, Emperador y Lloc Nou de la Corona, cuya población total en 2011 era de 4.187 habitantes y constituía el 0,16\% del total del AMV. Por el contrario, se han retenido como casos de estudio los municipios de Canet d'En Berenguer, Náquera, la Eliana y Massalfassar, que carecían de información en 2011 en una o dos de las 26 variables utilizadas, dado que en todos los casos se trataba de información de carácter secundario y no sustancial para la clasificación. Para estos cuatro municipios la información ausente se ha sustituido por el valor medio de la variable en el conjunto de todos los casos de estudio. 
La unidad territorial básica utilizada para el análisis en la mayor parte del área ha sido el municipio, debido a la dificultad de obtener información a escala más detallada para 2011 en el caso de los municipios de menor tamaño debido a razones de secreto estadístico y error muestral. Sólo para la ciudad de Valencia se ha optado por tratar la información a escala de distrito censal debido a que su tamaño demográfico así lo permitía, y de esta forma era posible tener en cuenta la diversidad interna de la propia ciudad. Por tanto, el número final de unidades territoriales es de 89, incluyendo 70 municipios y 19 distritos de la ciudad de Valencia.

El Análisis de Componentes Principales (ACP) o Factorial permite determinar los factores subyacentes comunes a grupos de variables que se encuentran parcial o totalmente asociadas entre sí. El objetivo es hallar una serie de factores que expliquen el máximo de varianza común de las variables originales. Se trata de una metodología ampliamente utilizada para la clasificación y análisis de la estructura interna de las áreas urbanas, si bien para que sea útil el resultado cuantitativo debe ser interpretado a la luz de un conocimiento detallado del territorio de análisis y de los procesos que le afectan. En el presente caso se ha aplicado un análisis factorial mediante el método de Componentes Principales a las 26 variables que se han seleccionado atendiendo al marco teórico y a los resultados de la matriz de correlaciones ${ }^{3}$. El tratamiento estadístico se ha realizado mediante el programa IBM SPSS Statistics versión 22C. El análisis ha dado resultados significativos de acuerdo con el test de Bartlett (nivel de significación inferior al 0'001 en los dos análisis realizados) y el índice Kaiser-Meyer-Olkin (KMO igual a 0'717 para 2001 y 0'694 en 2011). El ACP se ha basado en la matriz de correlaciones, $y$, para facilitar la interpretación de los resultados, se ha aplicado una rotación a la matriz mediante el método VARIMAX.

El análisis del gráfico de sedimentación ha aconsejado retener los cuatro primeros componentes principales en la primera fecha de referencia (2001-2002), y los seis primeros en la segunda (2011-2012); estos componentes suman respectivamente el 70’6\% y 76’2\% de la varianza total de los valores iniciales. Las tablas de pesos o saturaciones factoriales (tablas 2 y 3 ) muestran las variables asociadas a cada uno de estos factores principales, y nos permiten interpretar el sentido de cada uno de ellos de acuerdo con los indicadores seleccionados para el análisis.

Una vez identificados los principales factores subyacentes a las desigualdades sociodemográficas existentes en el área, se ha aplicado un análisis cluster o de agrupamiento a los componentes extraídos a partir del análisis factorial, estableciendo una tipología de las unidades territoriales que facilite el análisis comparativo. Los métodos cluster son métodos heurísticos que están diseñados para crear grupos homogéneos de casos o entidades y permiten desarrollar una tipología o clasificación, así como analizar su evolución temporal por comparación entre los distintos periodos de referencia. La estrategia del análisis cluster es buscar la estructura de los datos que no es fácilmente aparente a través de inspección visual (Moreno y García López, 2011).

3 Todas las variables retenidas son indicativas de una dimensión relevante dentro de los procesos urbanos, y mantienen correlaciones significativas a un nivel de significación del 0’05 (dos colas) con al menos otras 11 variables en 2001 y al menos 6 en 2011 . 
Como índice de disimilaridad se ha utilizado la distancia euclidiana al cuadrado, realizando el agrupamiento mediante un proceso aglomerativo jerárquico, que permite explorar más fácilmente la estructura interna de los datos (Aldenderfer y Blashfield, 1986). Este método parte de tantos grupos como casos de estudio, para ir agrupando sucesivamente los casos que presentan valores de disimilaridad más reducidos. Tras algunas pruebas, se decidió aplicar el método de enlace completo, que es uno de los más rigurosos (un candidato debe tener un cierto nivel de similaridad con todos los miembros del grupo), y tiende a formar agrupamientos relativamente compactos compuestos por casos altamente similares, facilitando así la detección de los grupos significativos.

Dado que las variables de clasificación proceden del análisis factorial y se encuentran por tanto ya estandarizadas, no se ha considerado necesario aplicar ningún tipo de transformación previa a los datos ni seleccionar otros índices de similaridad no afectados por el tamaño que podrían ser más apropiados en otras circunstancias.

\section{RESULTADOS: LA ESTRUCTURA INTERNA DEL AMV}

\section{III.1. Evolución reciente del Área Metropolitana de Valencia}

El Área Metropolitana de Valencia (AMV) tiene su origen, como otras áreas metropolitanas españolas, en la transformación estructural generada por el proceso de industrialización a partir de los años 1960. A partir de este momento, atraviesa varias etapas. En la primera, hasta 1975, el crecimiento demográfico y económico, vinculado al proceso industrializador, se concentra en el municipio central y en los municipios del arco suroeste. A partir de esta fecha, y hasta aproximadamente 1980, se desarrolla el eje noroeste hacia el Rincón de Ademuz, debido al crecimiento industrial, residencial y terciario favorecido por la mejora de las infraestructuras de comunicación. La tercera etapa se inicia a partir de mediados de los años 1980, y se caracteriza por la aceleración de los procesos de descentralización residencial y de las actividades económicas. En esta fase se produce la consolidación del eje noroeste y la integración en las dinámicas metropolitanas de algunos municipios de l'Horta Nord, así como de otros municipios más alejados ubicados sobre la autovía a Madrid al oeste y el corredor Valencia-Xàtiva al sur (Sorribes, 1997, pp. 239-241, y 1999, pp. 230-232). A finales de siglo XX, la estructura de usos del suelo del área se caracteriza por la fuerte especialización terciaria de la ciudad de Valencia, mientras que tanto la primera como la segunda corona metropolitana mantienen una especialización relativa netamente industrial, pese a la creciente expansión de los usos residenciales y terciarios.

Durante el primer decenio del siglo XXI, el Área Metropolitana de Valencia ha experimentado varios procesos territoriales de gran importancia. En primer lugar, se ha producido un fuerte incremento de la urbanización periférica; de acuerdo con datos de CORINE, entre los años 2000 y 2006 casi 46 millones de $\mathrm{m}^{2}$ se han transformado en suelo urbanizado en el AMV, sea como tejido urbano continuo (15\%), discontinuo (7'6\%), industrial y comercial (24’1\%) o como zonas en construcción (53’3\%). La mayor parte de este crecimiento ha tenido lugar en los municipios periféricos, en especial en la segunda corona metropolitana, y dentro de ésta, en el eje noroeste (Pitarch, Albertos y Cañizares, 2010, p. 533). 
Esta aceleración del proceso de urbanización difusa en la segunda corona metropolitana se ha visto favorecido por cambios en la oferta de infraestructuras de transporte tanto de nuevos ejes viarios de alta capacidad (CV-35 en la periferia Noroeste metropolitana, CV-30 y Ronda Norte de la Ciudad de Valencia) como de nuevas líneas de transporte ferroviario a partir de la expansión de la red de Metrovalencia (Pitarch, Albertos y Cañizares, 2010, p. 534). Los nuevos crecimientos suburbanos, junto con la mejora de las comunicaciones, han causado un importante aumento de la movilidad y la consolidación de una extensa área de mercado local de trabajo que en 2001 ya sumaba la tercera parte de la población de la región (Salom y Casado, 2007).

Un segundo proceso de gran importancia es el enorme aumento de la afluencia de población inmigrante, procedente en particular de países no comunitarios, que ha sido especialmente significativa tanto en la ciudad de Valencia (entre 2001 y 2011, los extranjeros pasaron de suponer el 5’2\% de la población total al 13'6\%\%) como en el conjunto metropolitano (del 3’8\% al 11'1 entre ambas fechas). En relación con la pauta geográfica de asentamiento de esta población en el ámbito metropolitano, es posible distinguir dos etapas. Durante la década de 1990, en la que la inmigración extracomunitaria era aún muy reducida, la población inmigrada se asentaba prioritariamente en los barrios populares del centro histórico, y en algunos otros enclaves dentro del casco urbano como Russafa y El Camí Fondo. Con el comienzo del presente siglo, se inicia una segunda fase en que, paralelamente al aumento del flujo migratorio, principalmente procedente de Ecuador y Colombia, se produce la extensión de su presencia a un mayor número de barrios de la ciudad, así como a núcleos obreros de la periferia. La relativamente amplia y extensa mezcla de inmigrantes y nativos que se da en la ciudad ha llevado a algunos investigadores a describir la situación más que como segregación, como una convivencia residencial multicultural, si bien muy desigualmente repartida por barrios (Torres, 2007, p. 82-83; p. 86). Esta imagen queda confirmada desde un punto de vista cuantitativo por Domínguez et al. (2010), quienes, a partir del cálculo de índices de segregación en el periodo comprendido entre 2000 y 2009, hallaron que la ciudad de Valencia mostraba los índices de segregación más reducidos de las ocho áreas metropolitanas españolas estudiadas, que, siguiendo la pauta que caracteriza a los países mediterráneos (Malheiros, 2002), eran a su vez menores que los hallados en otros entornos territoriales. También el ritmo de descenso de la segregación entre 2000 y 2009 fue superior en Valencia al del resto de áreas urbanas estudiadas.

El tercer gran proceso desde un punto de vista territorial es el impacto de la crisis económica, especialmente notable a partir de 2008. El AMV, que había mantenido durante el periodo 2001-2008 una evolución del mercado de trabajo más favorable que la media regio$\mathrm{nal}^{4}$, evoluciona peor que la media a partir esta fecha; por primera vez en el siglo la tasa media de crecimiento anual del paro registrado en el AMV supera la media de la Comunidad Valenciana, alcanzando el 34,4\% de incremento frente al 32,5\% del conjunto regional, lo que supone la inversión de una tendencia que se había mantenido durante décadas. Este deterioro del mercado de trabajo ha sido importante en todos los sectores económicos (Salom y Albertos, 2014, p. 543-545).

4 Entre 2001 y 2006, el incremento de la tasa de paro fue de sólo un 1,75\% en el AMV, frente al 7’24\% del conjunto de la Comunidad Valenciana; entre 2006 y 2008, esta cifra aumenta hasta el 14'02\%, aún por debajo del 16 '55\% de media regional (Salom y Albertos, 2014). 
Desde el punto de vista de las desigualdades internas, el impacto de la crisis económica ha sido doble: por un lado, desde un punto de vista territorial, ha tendido a homogeneizar a peor la situación de los distintos municipios tanto en relación con el mercado de trabajo como en cuanto a la capacidad adquisitiva y el índice de actividad económica. Por otro lado, si tenemos en cuenta la distribución de la población, los efectos negativos de la crisis se han concentrado en determinados municipios de la primera corona metropolitana, en particular los ubicados en l'Horta Nord y Sud, aumentando así las desigualdades internas medidas a través del índice de Gini ${ }^{5}$ (Salom, J. y Pitarch, M.D., 2014).

La tabla 2 resume la evolución de las variables analizadas en este artículo durante el periodo intercensal. En ella se muestra el incremento del valor medio y global de variables tales como el nivel educativo de la población, la tasa de paro, las viviendas hipotecadas y en régimen de alquiler, y el porcentaje de extranjeros sobre la población total (valores sombreados en la tabla en las columnas correspondientes), así como la reducción de las disparidades entre unidades territoriales en relación con los indicadores más estrechamente relacionados con la crisis económica (tasa de paro, porcentaje de viviendas hipotecadas) y la expansión de la inmigración (valores sombreados en las columnas correspondientes al coeficiente de variación).

Tabla 2

ESTADÍSTICOS DESCRIPTIVOS DE LAS VARIABLES UTILIZADAS EN EL ANÁLISIS

\begin{tabular}{|c|c|c|c|c|c|c|}
\hline \multirow{3}{*}{ VARIABLE (\%) } & \multicolumn{4}{|c|}{ UNIDADES TERRITORIALES } & \multirow{2}{*}{\multicolumn{2}{|c|}{$\begin{array}{c}\text { CONJUNTO } \\
\text { METROPOLITANO }\end{array}$}} \\
\hline & \multicolumn{2}{|c|}{$\begin{array}{l}\text { VALOR } \\
\text { MEDIO }\end{array}$} & \multicolumn{2}{|c|}{$\begin{array}{l}\text { COEFICIENTE } \\
\text { VARIACIÓN }\end{array}$} & & \\
\hline & 2001 & 2011 & 2001 & 2011 & 2001 & 2011 \\
\hline Analfabetos & 2,2 & 1,4 & 0,4 & 0,6 & 2,3 & 1,4 \\
\hline Población sin estudios & 11,5 & 8,1 & 0,3 & 0,3 & 11,2 & 7,8 \\
\hline Pob. con estudios de primer grado & 24,5 & 14,1 & 0,3 & 0,2 & 22,2 & 13,7 \\
\hline Pob. con estudios de tercer grado & 13,0 & 20,0 & 0,6 & 0,5 & 15,2 & 22,1 \\
\hline Tasa de paro & 11,1 & 30,0 & 0,2 & 0,1 & 12,8 & 30,6 \\
\hline Ocupados en la Agricultura & 5,8 & 3,7 & 0,9 & 0,9 & 3,4 & 2,7 \\
\hline Ocupados en la Industria & 22,8 & 16,3 & 0,3 & 0,3 & 21,5 & 14,6 \\
\hline Ocupados en Servicios & 59,6 & 72,7 & 0,2 & 0,1 & 64,7 & 76,3 \\
\hline Viviendas hipotecadas & 26,1 & 40,3 & 0,3 & 0,2 & 27,7 & 37,9 \\
\hline Vivienda en régimen de alquiler & 7,0 & 9,1 & 0,6 & 0,5 & 9,2 & 11,2 \\
\hline
\end{tabular}

5 El índice de Gini de la distribución del paro registrado en relación con la población ha pasado del 0’77 al 0'79 entre 2008 y 2012; mientras que la cuota de mercado y el índice de actividad económica han pasado respectivamente del 0'76 al 0'78 y del 0'73 al 0’75 entre 2003 y 2012 (Salom, J. y Pitarch, M.D., 2014, p. 562-563) 


\begin{tabular}{|l|r|r|r|r|r|r|} 
Viviendas sin calefacción & 73,7 & 64,5 & 0,1 & 0,2 & 73,2 & 65,9 \\
Vivienda anterior a 1960 & 22,7 & 16,0 & 0,4 & 0,5 & 23,6 & 17,8 \\
Vivienda construida 1960-2001 & 73,4 & 59,2 & 0,1 & 0,2 & 72,9 & 62,9 \\
Antigüedad media de la vivienda & 32,8 & 33,5 & 0,2 & 0,2 & 32,7 & 31,5 \\
Superficie de la vivienda $<90 \mathrm{~m}^{2}$ & 43,6 & 44,7 & 0,3 & 0,3 & 49,4 & 50,5 \\
Viviendas de 120 m². o más & 17,6 & 20,6 & 0,6 & 0,5 & 13,4 & 15,0 \\
Hogares unipersonales & 20,1 & 23,1 & 0,2 & 0,2 & 20,8 & 25,0 \\
Hogares con cinco o más personas & 9,3 & 4,9 & 0,2 & 0,3 & 9,1 & 4,9 \\
Tamaño medio del hogar & 2,8 & 2,5 & 0,0 & 0,1 & 2,7 & 2,6 \\
Población menor de 15 años & 13,8 & 15,6 & 0,1 & 0,1 & 13,8 & 15,3 \\
Población entre 15 y 29 años & 22,9 & 16,3 & 0,1 & 0,1 & 23,0 & 16,4 \\
Mayores de 65 años & 15,6 & 16,3 & 0,2 & 0,2 & 15,7 & 16,8 \\
Porcentaje de Extranjeros & 3,0 & 9,4 & 0,5 & 0,4 & 3,8 & 11,1 \\
Extranjeros procedentes de la U.E. & 20,5 & 43,8 & 0,7 & 0,4 & 14,1 & 36,1 \\
Extranjeros procedentes de África & 19,3 & 15,5 & 0,7 & 0,6 & 18,0 & 15,7 \\
Extranjeros proc. de América & 35,8 & 28,6 & 0,4 & 0,4 & 46,9 & 33,5 \\
\hline
\end{tabular}

Fuente: Censos de Población, 2001 y 2011; Padrones de Población, 2002 y 2012. Elaboración propia.

Así pues, los efectos territoriales de la crisis se han sobreimpuesto a las tendencias seculares de relocalización y descentralización residencial y de las actividades económicas, dando como resultado la modificación de la estructura interna metropolitana. En las páginas siguientes estudiaremos cómo se ha concretado este cambio en las características sociodemográficas de la población residente.

\section{III.2. Los componentes de la desigualdad urbana (2001-2011)}

Los resultados del análisis factorial aplicado a la información de la primera fecha de referencia (2001-2002) han aconsejado retener los primeros cuatro componentes principales, que permiten explicar un 70'58\% de la varianza total de los valores iniciales y un 56'82\% de la varianza tras aplicar la rotación de la matriz. La tabla de pesos o saturaciones factoriales (tabla 3 ) muestra las variables asociadas a cada uno de estos cuatro factores principales, y nos permite interpretar el sentido de cada uno de ellos de acuerdo con los indicadores seleccionados para el análisis. A partir de estas variables, se han definido estos cuatro componentes respectivamente como indicadores de centralidad urbana (componente 1), bajo nivel socioeconómico (componente 2), ciudad consolidada (componente 3), y ciclo de vidadinámica urbanística (componente 4), tal y como se detalla a continuación. 
Tabla 3

MATRICES FACTORIALES PARA 2001-2002 CON LAS VARIABLES ASOCIADAS A LOS CUATRO PRIMEROS COMPONENTES PRINCIPALES

\begin{tabular}{|c|c|c|c|c|}
\hline \multirow{2}{*}{ VARIABLE } & \multicolumn{4}{|c|}{ PESO FACTORIAL } \\
\hline & FACTOR 1 & FACTOR 2 & FACTOR 3 & FACTOR 4 \\
\hline Vivienda en régimen de alquiler & ,760 & 273 & ,268 & \\
\hline Porcentaje de Extranjeros & ,758 & ,242 & &,- 107 \\
\hline Ocupados en Servicios & ,708 & ,177 & 124 &,- 142 \\
\hline Población con estudios de tercer grado & 696 &,- 103 &, 152 & \\
\hline Hogares unipersonales & ,488 & & 244 &,- 739 \\
\hline Mayores de 65 años & ,304 & & & \\
\hline Extranjeros procedentes de América & 270 & ,737 & , 140 & \\
\hline Tasa de paro & ,253 & ,726 & & \\
\hline Hogares con cinco o más personas & ,206 &,- 145 & & ,904 \\
\hline Viviendas de 120 m. o más & , 179 &,- 755 & & ,307 \\
\hline Antigüedad media de la vivienda &, 159 & ,167 & 884 &,- 237 \\
\hline Extranjeros procedentes de África &,- 126 & & & \\
\hline Ocupados en la Agricultura &,- 161 &,- 442 &,- 149 & \\
\hline Analfabetos &,- 212 & ,258 & & ,191 \\
\hline Tamaño medio del hogar &,- 248 & &,- 220 & ,908 \\
\hline Población sin estudios &,- 281 & &,- 116 & \\
\hline Población con estudios de primer grado &,- 470 & & & \\
\hline Viviendas sin calefacción &,- 476 & ,347 & &,- 222 \\
\hline Población entre 15 y 29 años &,- 526 & ,442 &,- 234 &, 377 \\
\hline Ocupados en la Industria &,- 796 & ,242 & & ,107 \\
\hline Superficie de la vivienda $<90 \mathrm{~m} 2$ & & ,750 & &,- 156 \\
\hline Población menor de 15 años & &,- 160 &,- 391 & ,643 \\
\hline Entranjeros procedentes de la U.E. & &,- 681 & & \\
\hline Vivienda anterior a 1960 & & &, 924 &,- 126 \\
\hline Viviendas hipotecadas & & &,- 508 & \\
\hline Vivienda construida a partir de 1960 & & &,- 949 & ,216 \\
\hline
\end{tabular}

Fuente: Instituto Nacional de Estadística: Censo 2001 y Padrón 2002. Elaboración propia.

1. Centralidad metropolitana: El primer componente, que supone un 27'09\% de la varianza total, aparece significativamente correlacionado con la ocupación en el sector servicios, la población con un nivel educativo y una edad media más elevados, los hogares unipersonales y la vivienda en régimen de alquiler. Por el contrario, se correlaciona negativamente con la ocupación en el sector industrial, la población joven y con un nivel educativo más reducido, y la vivienda mal equipada. También está asociado a un alto porcentaje de población extranjera, aunque no específicamente extracomunitaria. Se trata pues de un componente que identifica claramente a los espacios urbanos centrales, caracterizados por la especialización en el sector servicios y por la presencia de población con un nivel socioeconómico más elevado. Los distritos centrales del municipio de Valencia, junto con el municipio de Rocafort, marcado por su carácter residencial de clase media y media alta, son las áreas que concentran los valores positivos más elevados.

2. Bajo nivel socioeconómico: El segundo componente, que supone un 19'6\% adicional de la varianza, se encuentra asociado positivamente con la tasa de desempleo, el porcen- 
taje de inmigrantes procedentes de países americanos, la población joven y analfabeta, y la vivienda de pequeño tamaño y mal equipada. Esto nos lleva a definirlo como un indicador de mala situación socioeconómica o de pobreza urbana. Los mayores valores se encuentran en los distritos periféricos de Valencia situados al noroeste y sudeste, y, en segundo lugar, en los municipios industriales (oeste y suroeste) de la primera corona metropolitana.

3. Ciudad consolidada: Por su parte, el tercer componente (16'8\% de la varianza) resume la información de las variables demográficas y de vivienda asociadas a los cascos urbanos: vivienda antigua y en régimen de alquiler, población adulta, y alta frecuencia de hogares unipersonales. Los valores más elevados se concentran en los distritos centrales del municipio de Valencia y en algunos pequeños municipios del continuo urbano como Massamagrell, Tavernes Blanques y Benetusser. También muestran valores positivos Sagunt y otros municipios ubicados al norte y al sur de la ciudad que incluyen barrios con estas características.

4. Demografía expansiva-Barrios nuevos: Finalmente, el cuarto componente (7'1\% de la varianza) presenta un carácter claramente demográfico, y está asociado a los barrios de expansión posterior a la década de 1960, con hogares de gran tamaño (cinco o más miembros), abundante población joven (niños y jóvenes hasta 29 años) y viviendas grandes. Los valores positivos se concentran en la corona metropolitana occidental, en especial en los municipios residenciales de eje Noroeste (Godella, Rocafort, La Eliana).

Estos resultados coinciden en términos generales con los obtenidos habitualmente en estudios similares en cuanto a la importancia de los componentes socioeconómico, demográfico y de ciclo de vida. En nuestro caso, el uso de una definición urbana de carácter funcional, que supone incorporar en el ámbito de estudio espacios fuera del continuo urbanizado pero vinculados funcionalmente, concede además un peso importante a aspectos tales como los niveles de centralidad y las distintas dinámicas constructivas.

Los resultados de este mismo análisis para el periodo 2011-2012 muestran diferencias bastante significativas. Un primer hecho a observar es que, para la fecha más reciente, el análisis del gráfico de sedimentación muestra la conveniencia de retener un mayor número de componentes (hasta seis) para realizar una correcta interpretación de la estructura metropolitana. Estos seis primeros componentes suman en conjunto un nivel de explicación sólo ligeramente superior al de los cuatro factores significativos para el año 2001-2002 (76’2\% frente a 70'6\%), lo que apunta a una mayor complejidad de los procesos subyacentes que articulan la estructura metropolitana, o al menos a un comportamiento más disperso de las variables consideradas.

De acuerdo con la tabla de pesos o saturaciones factoriales (tabla 4), estos seis factores se han interpretado como:

1. Bajo nivel socioeconómico: El componente asociado a condiciones socioeconómicas negativas se constituye ahora en el principal factor explicativo de la estructura territorial del área, suponiendo un 24'4\% de la varianza explicada. Aunque las variables más significativas de este componente siguen siendo, como en el año 2001, la tasa de paro y las malas condiciones de la vivienda, el impacto generalizado de la crisis económica y el proceso de dispersión geográfica de la población inmigrante no comunitaria ha hecho desaparecer la asociación de este factor con el peso de la población procedente de países americanos ${ }^{6}$, mientras que aparece una correlación muy intensa con los niveles educativos y, en menor medida, con el porcentaje de ocupados en el sector industrial.

6 Sí que se mantiene, por el contrario, cierta asociación con el porcentaje de personas nacidas en países africanos. 
Tabla 4

MATRICES FACTORIALES PARA 2011-2012 CON LAS VARIABLES ASOCIADAS A LOS SEIS PRIMEROS COMPONENTES PRINCIPALES

\begin{tabular}{|c|c|c|c|c|c|c|}
\hline \multirow{2}{*}{ VARIABLE } & \multicolumn{6}{|c|}{ PESO FACTORIAL } \\
\hline & FACTOR 1 & FACTOR 2 & FACTOR 3 & FACTOR 4 & FACTOR 5 & FACTOR 6 \\
\hline Población sin estudios & ,828 & &,- 175 &,- 205 & &, 110 \\
\hline Viviendas sin calefacción & 813 & ,197 & ,130 & , 109 &,- 272 & \\
\hline Tasa de paro & ,785 & & & ,151 & ,188 & ,179 \\
\hline Población con estudios de primer grado & ,770 & , 166 &,- 140 &,- 180 &,- 179 & , 150 \\
\hline Analfabetos & ,728 & & & & & \\
\hline Extranjeros procedentes de África & ,658 & ,161 & ,245 &,- 186 & &,- 163 \\
\hline Superficie de la vivienda $<90 \mathrm{~m} 2$ &, 588 & & ,584 & ,420 & & \\
\hline Ocupados en la Industria &, 501 &,- 256 &,- 191 &,- 480 & & \\
\hline Ocupados en la Agricultura & ,224 & ,185 &,- 594 &,- 333 &,- 327 & , 175 \\
\hline Población entre 15 y 29 años & ,184 & &,- 125 & ,173 & ,262 & ,613 \\
\hline Vivienda construida entre 1960 y 2001 & ,130 & & ,292 & ,105 & & ,809 \\
\hline Población menor de 15 años &,- 126 &,- 765 & &,- 168 & ,233 &,- 132 \\
\hline Hogares con cinco o más personas &,- 149 & & & & ,834 & 167 \\
\hline Extranjeros procedentes de América &,- 157 & ,113 & ,841 & &,- 166 & , 160 \\
\hline Hogares unipersonales &,- 169 & ,551 & ,113 & ,439 &,- 503 & \\
\hline Extranjeros procedentes de la U.E. &,- 244 &,- 197 &,- 876 & & ,111 & \\
\hline Vivienda en régimen de alquiler &,- 322 & ,316 & ,262 &, 595 &,- 121 & ,192 \\
\hline Ocupados en Servicios &,- 511 & ,150 &, 527 & ,491 & ,216 & \\
\hline Viviendas de $120 \mathrm{~m}$. o más &,- 598 &,- 143 &,- 532 &,- 433 & ,228 & \\
\hline Población con estudios de tercer grado &,- 836 & ,205 & ,179 & ,225 & &,- 184 \\
\hline Antigüedad media de la vivienda & & ,839 & ,183 & ,322 & ,106 &,- 162 \\
\hline Mayores de 65 años & & ,838 & &,- 137 & & \\
\hline Vivienda anterior a 1960 & & ,745 & & ,210 & &,- 484 \\
\hline Porcentaje de Extranjeros & & ,204 & & ,821 & & ,179 \\
\hline Tamaño medio del hogar & &,- 477 &,- 123 &,- 420 & ,633 & ,153 \\
\hline Viviendas hipotecadas & &,- 871 & &,- 158 & &,- 319 \\
\hline
\end{tabular}

Fuente: Instituto Nacional de Estadística: Censo 2011 y Padrón 2012. Elaboración propia.

La pauta territorial, más contrastada que en el año 2001, es también distinta: Los valores más elevados se concentran ahora en los municipios situados en el sector sur y suroeste de la primera corona metropolitana, entre los que destacan especialmente los municipios de Manises y Quart de Poblet, así como en algunos municipios de la periferia metropolitana de las comarcas de Camp de Túria y Hoya de Buñol. Por el contrario, los valores más reducidos se ubican en los distritos del municipio de Valencia y a lo largo del eje noroeste de la primera corona metropolitana. El mapa 1 muestra los cambios experimentados en la distribución territorial de este componente en el periodo intercensal. Los valores negativos, asociados con una mejora de la posición relativa de los municipios en el contexto socioeconómico metropolitano, se concentran en la ciudad de Valencia, municipios próximos de carácter residencial (Burjassot Godella, Rocafort, La Eliana, etc.), y Almussafes; mientras que los valores positivos, que indican un empeoramiento de la posición relativa, se localizan en la periferia metropolitana, en los municipios de Quart de Poblet y Manises, y en l'Horta Sud. 
Es evidente, pues, que la crisis económica ha tenido como principal consecuencia territorial un agravamiento de las desigualdades socioeconómicas en el interior del Área Metropolitana con un patrón geográfico muy específico.

Mapa 1

EVOLUCIÓN DEL COMPONENTE "BAJO NIVEL SOCIOECONÓMICO" ENTRE 2001 Y 2011: PUNTUACIÓN FACTORIAL EN 2011 DEL COMPONENTE 1 MENOS PUNTUACIÓN FACTORIAL EN 2001 DEL COMPONENTE 27.

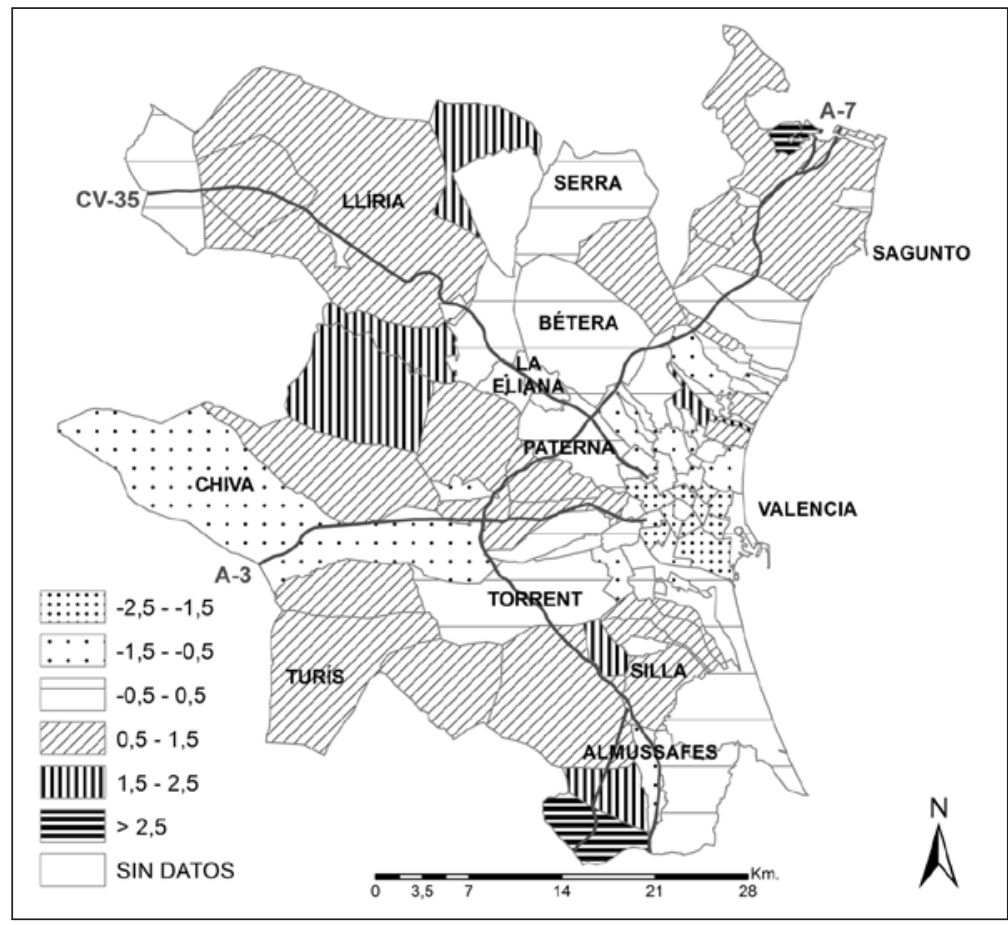

Fuente: Instituto Nacional de Estadística: Censo 2001 y Padrón 2002. Elaboración propia.

2. Ciudad consolidada: Este segundo componente, que tiene un peso muy similar al primero (23'1\% de la varianza explicada), coincide en términos generales con el definido con este mismo nombre para el año 2001. Se asocia pues positivamente con los indicadores relacionados con la antigüedad de la vivienda, la población mayor de 65 años, la presencia de familias unipersonales, y la vivienda en régimen de alquiler, mientras que presenta una asociación negativa con la población joven, la vivienda hipotecada, y el tamaño medio del hogar, indicadores característicos de los barrios de crecimiento reciente y que acogen a las familias jóvenes con hijos. Su pauta territorial no ha experimentado grandes cambios en relación con la existente en 2001.

7 Los valores negativos indican un aumento del nivel socioeconómico relativo de la unidad territorial entre ambas fechas, mientras que los valores positivos indican un empeoramiento de la situación socioeconómica de la unidad territorial en relación con la media del AMV. 
3. Población inmigrante no comunitaria: Este tercer componente, que resume un $11 \%$ de varianza explicada, está asociado al proceso de segregación de la inmigración por lugares de procedencia y condición socioeconómica. Presenta una correlación positiva con la inmigración procedente de países americanos, y una correlación negativa con la inmigración procedente de países de la Unión Europea. También, aunque de manera menos significativa, se encuentra asociado al porcentaje de viviendas pequeñas y de ocupados en el sector servicios. Los mayores valores se concentran en la ciudad de Valencia salvo el distrito de Ciutat Vella, y en los municipios de la primera corona metropolitana, en particular en aquellos ubicados al oeste y sur de la ciudad.

4. Porcentaje de población extranjera: A diferencia del factor anterior, este cuarto componente, que supone un 7’ $47 \%$ de la varianza explicada, está básicamente relacionado con el peso demográfico que representan los extranjeros, sean procedentes de países comunitarios o no comunitarios, sobre la población total, aunque recoge también, de manera secundaria, algunas de las variables asociadas en 2001 con la centralidad urbana, tales como el peso del sector servicios y el porcentaje de vivienda en alquiler. Los valores positivos de este componente se concentran, con escasas excepciones, en el municipio de Valencia.

5. Demografía expansiva: El componente quinto (5'67\% de la varianza) recoge las variables demográficas asociadas con el ciclo de vida: hogares con un elevado número medio de miembros y un elevado porcentaje de niños y jóvenes. Los valores más elevados se ubican en los municipios del norte y noroeste de la conurbación valenciana que han experimentado un mayor crecimiento suburbano (Godella, Rocafort, Alfara del Patriarca, Foios, Albalat del Sorells), mientras que encontramos valores negativos en los distritos de la ciudad central y en los municipios periféricos del área metropolitana. El modelo territorial general es bastante similar al de 2001 .

6. Barrios nuevos: Por último, el componente sexto (4'51\% de la varianza), se encuentra asociado a la presencia de vivienda nueva y en alquiler y a la población joven, siendo estos indicadores representativos de los barrios de crecimiento urbanístico reciente. Geográficamente, los valores positivos se concentran en municipios muy concretos localizados tanto en el municipio de Valencia (distritos periféricos del nordeste), como en el eje noroeste.

En resumen, los principales cambios detectados en relación con los factores principales que explican la estructura residencial del AMV entre 2001 y 2011 son los siguientes:

- Un aumento de la complejidad o, al menos, de la dispersión del comportamiento de las variables consideradas, que se explica por la introducción de nuevos factores asociados a procesos crecientemente influyentes en la estructura metropolitana.

- El aumento de la importancia del factor socioeconómico y el retroceso de los factores demográficos y urbanísticos como elementos explicativos de la estructura interna metropolitana.

- La irrupción de nuevos factores asociados a la entrada de población inmigrante, relacionados tanto con su peso demográfico relativo, como con su procedencia geográfica (comunitaria vs no comunitaria), que pasan a jugar un papel explicativo importante en los desequilibrios internos.

- La tendencia a la disociación de las variables correspondientes al ciclo de vida de la población residente con las asociadas a la dinámica urbanística, proceso que podemos relacionar con la existencia de proyectos urbanísticos muy focalizados y no relacionados con el crecimiento demográfico. 


\section{III.3. Cambios en la tipología sociodemográfica del AMV (2001-2011)}

Los análisis cluster realizados sobre los componentes principales hallados a partir de los datos de 2001 y 2011 (cuatro y seis respectivamente) han permitido elaborar una tipología de unidades territoriales para cada una de las fechas indicadas. La inspección de los dendogramas y los gráficos de coeficientes de fusión (Aldenderfer y Blashfield, 1986) ha determinado la subdivisión final en ocho grupos para 2001 y de nueve en 2011. La localización geográfica de estos grupos se representa en las figuras 2 y 3, y sus características principales aparecen resumidas en las tablas 5 y 6 .

Tal y como puede verse en la figura 2, la localización geográfica de los grupos identificados en 2001 dibuja un patrón general en círculos concéntricos a partir del casco histórico de Valencia, pudiendo diferenciarse tres anillos sucesivos:

1. Centro metropolitano: Está compuesto por tres grupos de unidades territoriales ubicadas en su mayoría en el municipio de Valencia, y caracterizadas por poseer los mayores valores de centralidad del área. Desde el núcleo central a la periferia, son las siguientes:

- Grupo 1: Está formado por los tres distritos centrales del municipio de Valencia (Ciutat Vella, Extramurs y L'Eixample), que se caracterizan por alcanzar los valores más elevados del área en los componentes 1 y 3 (centralidad urbana y ciudad consolidada), y valores negativos en los componentes 2 y 4 (baja condición socioeconómica y demografía expansiva-barrios nuevos).

- Grupo 2: Incluye un segundo anillo formado por el resto de distritos de Valencia, salvo los más periféricos y el Plá del Real (distrito 6). Estas unidades espaciales tienen también valores elevados de centralidad, pero, a diferencia del grupo anterior, son áreas receptoras de inmigración y presentan condiciones socioeconómicas negativas.

- Grupo 3: Incluye el distrito del Plá del Real en Valencia y los municipios conurbados de Godella y Rocafort, tres zonas que se caracterizan por su condición de áreas residenciales de clase media y alta. Son unidades territoriales con un alto nivel de centralidad, una condición socioeconómica muy positiva y una dinámica demográfica expansiva.

2. Primera corona metropolitana: Incluye los distritos periféricos del municipio de Valencia y los municipios de la inmediata comarca de l'Horta, que comparten un escaso nivel de centralidad, una situación socioeconómica negativa y una fuerte dinámica demográfica y constructiva.

- Grupo 4: Los municipios de este grupo se encuentran localizados mayoritariamente en la sección occidental de la primera corona metropolitana y se corresponden con la principal área receptora de la corriente inmigratoria de los años 1960. Se caracterizan por una peor situación socioeconómica y una dinámica demográfica más expansiva que el resto de la primera corona.

- Grupo 5: Este grupo incluye la mayor parte de los municipios ubicados al norte y al sur del centro urbano. Estás áreas son las que experimentaron un menor crecimiento 

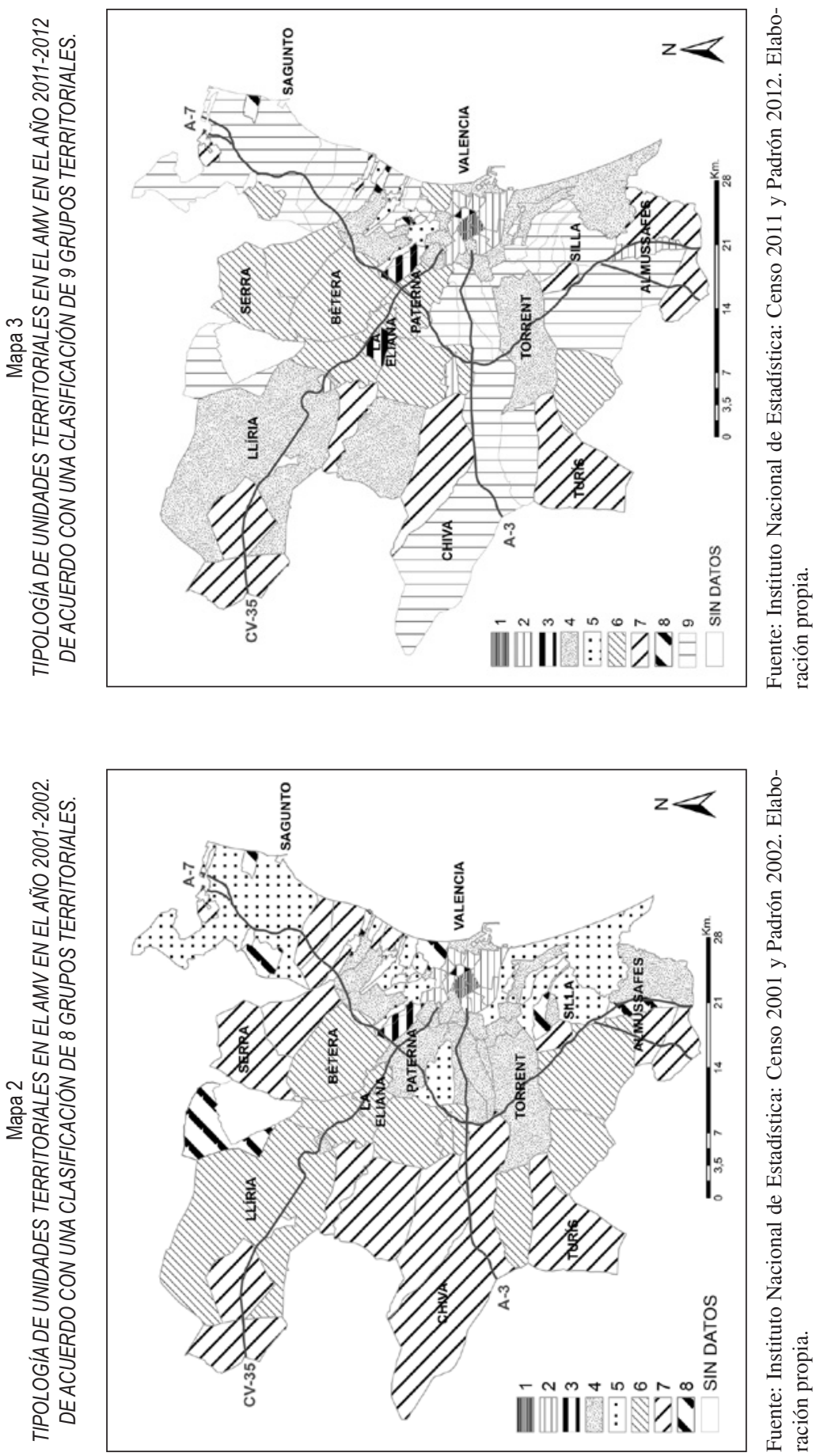
demográfico por inmigración en los años 1960, por lo que tienen una estructura de edades y una tipología de vivienda más envejecidas, pero también un menor nivel de pobreza urbana.

Tabla 5

PROMEDIO DE LAS PUNTUACIONES FACTORIALES DE LOS CLÚSTER TERRITORIALES, 2001-2002

\begin{tabular}{|c|c|c|c|c|c|c|}
\hline $\begin{array}{l}\text { LOCALIZACIÓN } \\
\text { GEOGRÁFICA }\end{array}$ & GRUPO & $\begin{array}{c}\text { No } \\
\text { MUNICIPIOS }\end{array}$ & $\begin{array}{c}\text { FACTOR } 1 \\
\text { CENTRALIDAD }\end{array}$ & $\begin{array}{c}\text { FACTOR } 2 \\
\text { BAJO NIVEL } \\
\text { SOCIOECONÓMICO }\end{array}$ & $\begin{array}{c}\text { FACTOR } 3 \\
\text { CIUDAD } \\
\text { CONSOLIDADA }\end{array}$ & $\begin{array}{c}\text { FACTOR } 4 \\
\text { CICLO DE VIDA - } \\
\text { NUEVOS BARRIOS }\end{array}$ \\
\hline \multirow{3}{*}{ CENTRO } & 1 & 3 & 2,60 & $-0,48$ & 2,32 & $-1,31$ \\
\hline & 2 & 10 & 1,12 & 1,41 & $-0,64$ & $-0,62$ \\
\hline & 3 & 3 & 1,51 & $-0,70$ & 0,56 & 2,43 \\
\hline \multirow{2}{*}{$\begin{array}{l}\text { PRIMERA } \\
\text { CORONA }\end{array}$} & 4 & 21 & $-0,27$ & 0,87 & $-0,08$ & 0,48 \\
\hline & 5 & 15 & $-0,87$ & 0,13 & 1,06 & 0,01 \\
\hline \multirow{2}{*}{$\begin{array}{c}\text { SEGUNDA } \\
\text { CORONA }\end{array}$} & 6 & 10 & 0,42 & $-1,06$ & $-1,11$ & 0,67 \\
\hline & 7 & 20 & $-0,24$ & $-0,82$ & 0,20 & $-0,17$ \\
\hline OTROS & 8 & 7 & $-0,60$ & $-0,54$ & $-1,35$ & $-1,53$ \\
\hline
\end{tabular}

Fuente: Instituto Nacional de Estadística: Censo 2001 y Padrón 2002. Elaboración propia.

3. Segunda corona metropolitana: Los municipios de esta segunda corona, con un grado desigual de integración en la dinámica metropolitana, tienen como única característica común una situación socioeconómica mejor que los ubicados en la primera corona metropolitana. En función del impacto de los procesos de suburbanización residencial, podemos distinguir entre:

- Grupo 6: Aglutina a los municipios de mayor carácter residencial, en su mayoría ubicados en el noroeste y suroeste del área metropolitana. Se caracterizan por presentar las condiciones socioeconómicas más favorables del área, un carácter urbano intermedio y la segunda dinámica demográfica más expansiva del AMV.

- Grupo 7: Incluye a los municipios más periféricos, no afectados por la inmigración y por tanto con niveles socioeconómicos más positivos que el centro y la primera corona metropolitana, pero a los que tampoco han alcanzado los procesos de descentralización residencial. Estos municipios se distinguen por su menor nivel urbano, el mayor envejecimiento de su población, y su carácter semi-rural.

4. Otros: El grupo 8 incluye siete municipios ubicados en distintos puntos del área metropolitana, caracterizados por una buena condición socioeconómica, un crecimiento urbanístico reciente no ligado al crecimiento demográfico, y escasa inmigración, con predominio de la población procedente de la Unión Europea. Aunque existen distintas situaciones dentro del grupo, la mayor parte de los casos parecen estar relacionados con fenómenos de urbanización de segunda residencia vinculada a grupos demográficos de mayor edad. Algunos de ellos presentan además un fuerte desarrollo industrial. 
La comparación de esta tipología con la resultante del análisis de los datos de 2011 (9 grupos) nos permite observar un cambio general en las pautas de distribución territorial, que es posible describir como la tendencia a la ruptura del modelo territorial de anillos concéntricos, y su sustitución por sectores radiales articulados por los principales ejes de comunicación, resultando en una pauta territorial de mayor complejidad. En este modelo, apenas apuntado en 2001 y ya claramente estructurado en 2011, el centro metropolitano y la primera corona mantienen la estructura concéntrica, mientras que la segunda corona adopta un patrón sectorial articulado en torno a los principales ejes de comunicaciones. De acuerdo con esta estructura, podemos agrupar los tipos hallados en:

\section{Centro metropolitano:}

- Grupo 1: Este grupo es idéntico al hallado en 2001, tanto en cuanto a las unidades territoriales que lo componen (distritos centrales del municipio de Valencia) como en sus características económicas, demográficas y constructivas. En relación con los nuevos componentes hallados en 2011, se caracteriza por mostrar elevados valores de población inmigrante, en particular procedente de países no comunitarios.

- Grupo 2: Se corresponde también en términos generales con el grupo 2 de 2001 (distritos del municipio de Valencia exteriores al primer cinturón de ronda), aunque incorpora los municipios de Almussafes, Benisanó y Loriguilla, y pierde algunos de sus componentes más periféricos, que pasan a incluirse en el grupo 6. Las diferencias más importantes en relación con la situación de principios de siglo son la mejora sustancial de su situación socioeconómica relativa, los elevados valores de inmigración no comunitaria, y la existencia de nuevos proyectos urbanísticos.

Tabla 6

PROMEDIO DE LAS PUNTUACIONES FACTORIALES DE LOS CLÚSTER DE UNIDADES TERRITORIALES DEL AMV PARA EL AÑO 2011-2012

\begin{tabular}{|c|c|c|c|c|c|c|c|c|c|}
\hline \multicolumn{2}{|c|}{$\begin{array}{l}\text { LOCALIZACIÓN } \\
\text { GEOGRÁFICA }\end{array}$} & GRUPO & $\begin{array}{c}\text { No } \\
\text { MUNICIPIOS }\end{array}$ & $\begin{array}{c}\text { FACTOR } 1 \\
\text { BAJO NIVEL } \\
\text { SOCIOECONÓMICO }\end{array}$ & $\begin{array}{c}\text { FACTOR } 2 \\
\text { CIUDAD } \\
\text { CONSOLIDADA }\end{array}$ & $\begin{array}{c}\text { FACTOR } 3 \\
\text { INMIGRACIÓN NO } \\
\text { COMUNITARIA }\end{array}$ & $\begin{array}{c}\text { FACTOR } 4 \\
\text { POBLACIÓN } \\
\text { EXTRANJERA }\end{array}$ & $\begin{array}{l}\text { FACTOR } 5 \\
\text { CICLO DE } \\
\text { VIDA }\end{array}$ & $\begin{array}{l}\text { FACTOR } 6 \\
\text { NUEVOS } \\
\text { BARRIOS }\end{array}$ \\
\hline \multirow{2}{*}{\multicolumn{2}{|c|}{ CENTRO }} & 1 & 3 & $-2,00$ & 2,37 & 0,41 & 1,19 & $-0,41$ & $-1,98$ \\
\hline & & 2 & 11 & $-0,67$ & 0,35 & 0,79 & 0,14 & $-0,59$ & 1,27 \\
\hline \multirow{2}{*}{\multicolumn{2}{|c|}{ PRIMERA CORONA }} & 4 & 16 & 0,64 & $-0,02$ & 0,08 & 0,98 & 0,55 & 0,20 \\
\hline & & 5 & 2 & $-0,07$ & 1,91 & $-0,17$ & $-2,48$ & 1,79 & $-0,63$ \\
\hline \multirow{4}{*}{ 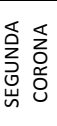 } & \multirow{2}{*}{\begin{tabular}{|c|} 
SECTOR \\
NOROESTE
\end{tabular}} & 6 & 10 & $-0,84$ & $-1,51$ & $-0,56$ & 0,39 & 0,23 & $-0,58$ \\
\hline & & 3 & 4 & $-2,16$ & 0,19 & 0,09 & $-0,24$ & 1,95 & 1,00 \\
\hline & \multirow{2}{*}{\begin{tabular}{|c|} 
EJES \\
RADIALES \\
\end{tabular}} & 8 & 4 & $-0,66$ & $-0,73$ & 0,20 & $-0,67$ & $-1,87$ & $-1,70$ \\
\hline & & 9 & 28 & 0,61 & $-0,16$ & 0,42 & $-0,50$ & 0,09 & $-0,22$ \\
\hline \multicolumn{2}{|c|}{ PERIFERIA } & 7 & 11 & 0,53 & 0,67 & $-1,65$ & $-0,17$ & $-0,87$ & 0,44 \\
\hline
\end{tabular}

Fuente: Instituto Nacional de Estadística: Censo 2011 y Padrón 2012. Elaboración propia.

\section{Primera corona metropolitana}

- Grupo 4: La mayoría de los municipios de este grupo forman un segundo anillo en torno a la ciudad de Valencia. Integra municipios que en el año 2001 formaban parte 
de los grupos 2 (periferia de Valencia), 5 (primera corona-norte y sur), y 4 (primera corona-oeste), y que en 2011 presentan uniformemente las características generales de este último grupo: altos niveles de pobreza urbana, elevados porcentajes de población inmigrante, y valores positivos y moderados de crecimiento demográfico y urbanístico.

- Grupo 5: Incluye el municipio de Albalat de Sorells y el distrito 17 de Valencia (Pobles del Nord) que, pese a ubicarse en esta primera corona metropolitana, no se han visto afectados de forma importante por los procesos que han producido la uniformización de situaciones del grupo anterior, y que por tanto han mantenido características relacionadas con su mayor carácter agrario: menores niveles de pobreza urbana, mayor nivel de envejecimiento, vivienda más antigua y tamaño medio del hogar mayor.

3. Segunda corona: De acuerdo con lo comentado anteriormente, diferenciamos entre el sector noroeste y los ejes radiales norte-sur y este-oeste articulados por las principales vías de comunicación.

a) Sector Noroeste:

- Grupo 6: Supone la consolidación del grupo 6 ya existente en 2001, que ahora se concentra geográficamente en el sector noroeste más próximo a la ciudad al tiempo que se amplía territorialmente hacia el nordeste. Los municipios de este grupo mantienen las características preexistentes en cuanto a nivel socioeconómico elevado, moderada inmigración de carácter mayoritariamente comunitario, y pujanza demográfica, aunque su crecimiento urbanístico se ha reducido en relación con la fecha anterior.

- Grupo 3: Está compuesto por los municipios de Rocafort, La Eliana y Godella, junto con el distrito 6 de Valencia, que conforman en realidad un subgrupo del grupo anterior con características más acentuadas en cuanto a nivel socioeconómico, consolidación urbana y dinámica demográfica. A diferencia del grupo anterior, han mantenido un fuerte crecimiento urbanístico en los últimos años.

b) Ejes radiales Norte/Sur-Este/Oeste:

- Grupo 9: Es el grupo que engloba a un mayor número de municipios (28), ubicados a los largo de dos ejes, uno norte-sur (desde Sagunt hasta Silla) y otro este-oeste (desde Quart de Poblet hasta Chiva) articulados en torno a las principales vías de comunicación metropolitanas. Las unidades territoriales de este grupo se caracterizan por una elevada pobreza urbana, inmigración reducida pero predominantemente no comunitaria, un factor demográfico moderadamente positivo, y ausencia de crecimiento urbanístico reciente. Además, en este grupo se incluyen aquellos municipios que presentan mayores porcentajes de población ocupada en el sector industrial. Los principales cambios experimentados desde 2001 por estos muni- 
cipios han sido un acusado deterioro de su situación socioeconómica, el aumento de la inmigración no comunitaria, y una cierta tendencia al envejecimiento de su población.

- Grupo 8: Incluye cuatro municipios situados en l'Horta Nord (Bonrepós i Mirambell, Canet, Massalfasar y Vinalesa) que se separan del grupo anterior por su mejor situación económica, y por una menor dinámica demográfica y urbanística. Se trata de unidades territoriales menos afectadas por los procesos de industrialización, que presentan por tanto un mayor porcentaje de activos ocupados en el sector servicios y menor peso de la inmigración no comunitaria ${ }^{8}$.

\section{Periferia}

- Grupo 7: Los municipios de este grupo poseen una localización intersticial entre los ejes transversales del AMV, por lo que se han visto afectados en menor medida por los procesos de industrialización y crecimiento demográfico, manteniendo un mayor peso del sector agrario y una estructura de edades más envejecida. En relación con 2001, estos municipios han experimentado también un fuerte empobrecimiento relativo, aunque no han sido afectados por la oleada inmigratoria, por lo que mantienen características demográficas similares a las existentes a principios de siglo. Es de destacar, sin embargo, la existencia de un cierto crecimiento urbanístico en los últimos años (valores medios positivos en el factor 6).

\section{CONCLUSIONES}

En primer lugar, y en relación con la metodología empleada, cabe concluir que el análisis de componentes principales seguido del análisis cluster, una técnica clásica en el estudio de la estructura sociodemográfica de las áreas residenciales en espacios urbanos, se ha revelado útil para estudiar la estructura interna y evolución reciente de un área urbana delimitada con criterios funcionales. Pese a las limitaciones derivadas de la ausencia de información para unidades territoriales pequeñas del Censo de Población y Viviendas de 2011, ha sido posible detectar la tipología sociodemográfica de las áreas residenciales metropolitanas y los factores subyacentes que la explican, así como los efectos territoriales de la irrupción de nuevos factores en el periodo 2001-2011.

La aplicación en este caso a un ámbito territorial más amplio de lo habitual, delimitado aquí por aspectos funcionales (principalmente movilidad residencia-trabajo) en vez de las usuales variables urbanísticas, ha dado un mayor peso en los resultados a factores diferenciales que inciden en la complementariedad funcional que se establece entre las unidades territoriales que componen el área de estudio, factores que aquí hemos etiquetado como centralidad urbana (ocupados en el sector servicios, población con niveles educativos superiores) y ciudad consolidada (antigüedad de la vivienda, población adulta), frente a los ya

8 Este grupo debe ser tomado con cierta precaución, ya que ha sido imposible conseguir información completa para dos de las unidades territoriales incluidas (Canet d'En Bereguer y Massalfassar) debido a su pequeño tamaño demográfico y al sistema de muestreo aplicado en el Censo de 2011, por lo que el valor específico ha tenido que ser sustituido por el valor medio del conjunto de unidades territoriales en dos de las variables utilizadas en el análisis cluster. 
clásicos relacionados con el estatus socioeconómico, familiar y étnico de la población, que no obstante quedan también adecuadamente recogidos en los resultados obtenidos.

Por otra parte, en relación con los cambios experimentados por la estructura sociodemográfica de los espacios residenciales urbanos del Área Metropolitana de Valencia a lo largo del último decenio, podemos concluir que durante el periodo de estudio se ha producido un cambio en el número y significado de los componentes principales explicativos de la segregación espacial en el AMV. En primer lugar, el componente asociado a condiciones socioeconómicas negativas se constituye ahora en el principal factor explicativo de la estructura territorial del área, suponiendo un 24 ' $4 \%$ de la varianza explicada, frente a sólo un 19 '6\% en 2001. Su patrón territorial también ha cambiado: La comparación de la distribución geográfica de este componente en 2001 y 2011 indica que se ha producido un aumento de los contrastes territoriales debido a la concentración geográfica tanto de los valores positivos como negativos, con una importante mejora relativa del centro y sector noroeste de la segunda corona metropolitana, frente al empeoramiento relativo de los municipios periféricos y de los situados al sur-suroeste de la ciudad de Valencia.

En segundo lugar, los factores asociados a la inmigración han ganado importancia en relación a los asociados con la dinámica demográfica y urbanística. Las variables relativas a la inmigración, asociadas en el año 2001 a indicadores definitorios de situaciones de pobreza urbana (tasa de paro, vivienda pequeña y mal equipada), conforman en 2011 una dimensión independiente, de modo que la inmigración, cualquiera que sea su procedencia geográfica, no está ya directamente relacionada con los barrios y municipios más empobrecidos del espacio urbano. En relación con lo anterior, la segregación geográfica por procedencia (inmigración comunitaria vs no comunitaria, en particular la procedente de países americanos) se constituye ahora en un componente de relativa importancia en la articulación del espacio urbano.

Finalmente, se observa que en estos diez años se ha producido una disociación geográfica entre las variables correspondientes al ciclo de vida de la población residente y las relacionadas con la dinámica urbanística, que en 2001 se presentaban asociadas a un mismo componente, y que en 2011 aparecen reflejadas en dos componentes distintos. Esto significa que el crecimiento urbanístico reciente no se corresponde necesariamente con una demografía expansiva, fenómeno que podemos relacionar con la existencia de proyectos urbanísticos muy focalizados y relacionados con factores externos al crecimiento demográfico del municipio.

Desde el punto de vista de la tipología sociodemográfica del espacio estudiado, en este periodo se ha producido un aumento de la complejidad del modelo territorial en anillos concéntricos que predominaba en el año 2001, dando paso a una pauta de sectores radiales marcadamente diferenciados, articulados por las principales vías de comunicación. Aunque este modelo apuntaba ya en 2001, especialmente en el área noroeste de la segunda corona metropolitana, en 2011 aparece ya claramente estructurado. El análisis de los grupos y su evolución temporal nos permite constatar la mejora experimentada por las unidades territoriales ubicadas en el municipio de Valencia y en el área noroeste de la segunda corona metropolitana, mientras que los municipios ubicados en la primera corona metropolitana, en el resto de la segunda, e incluso en el área periférica, han experimentado un empobrecimiento generalizado. 
En resumen, y aunque es difícil aislar los efectos de los distintos procesos experimentados por el Área Metropolitana de Valencia a lo largo del periodo estudiado (aumento de la integración funcional, entrada de nuevos flujos migratorios y crisis económica), podemos concluir que el resultado territorial global se caracteriza por un aumento de la polarización geográfica y de los contrastes sociodemográficos internos, lo que, a tenor de las consideraciones hechas en la primera parte de este artículo, sin duda tendrá importantes repercusiones sociales a medio y largo plazo.

\section{BIBLIOGRAFÍA}

ALDENDERFER, M.S. Y BLASHFIELD, R.K. (1986): Cluster Analysis, Sage University, London.

BAYONA, J. (2007): “La segregación residencial de la población extranjera en Barcelona: ¿Una segregación fragmentada?”. Scripta Nova. Revista Electrónica de Geografía y Ciencias Sociales. Vol. XI, núm. 235.

BELLET, C. (2007): "Los espacios residenciales de tipo privativo y la construcción de la nueva ciudad: visiones de Privatopía”. Scripta Nova. Revista Electrónica de Geografía y Ciencias Sociales. Vol. XI, núm. 245 (08).

BURGUESS, E. (1925): “The Growth of the City: An Introduction to a Research Project”, en R.Park, E.Burguess, y R.Mckenzie: The City, Chicago, University of Chicago Press.

DEL PINO, J.A. (2001): "La estructura social urbana de Málaga. Acercamiento a la estructura social urbana a partir de los datos censales sobre condición socioeconómica». Cuadernos de CC.EE. y EE, $\mathrm{n}^{\circ} 41,139-159$.

DÍAZ, I. (2010): "Cambios en la geografía social de Sevilla". Cuadernos Geográficos, no 46 (1), 139-161

DOMÍNGUEZ MUJICA, J; PARREÑO CASTELLANO, J.M., Y DÍAZ HERNÁNDEZ, R. (2010): "Inmigración y ciudad en España: Integración versus segregación socioterritoriales". Scripta Nova. Revista Electrónica de Geografía y Ciencias Sociales, Vol. XIV, núm. 331 (50).

ECHAZARRA, A. (2010): "Segregación residencial de los extranjeros en el área metropolitana de Madrid. Un análisis cuantitativo». Revista Internacional de Sociología, Vol. 68 (1), 165-197.

FERIA, J.M. (2008): “Un ensayo metodológico de definición de las áreas metropolitanas en España a partir de la variable residencia-trabajo". Investigaciones Geográficas, $\mathrm{n}^{\circ}$ 46, pp, 49-68.

FUNDACIÓN FOESSA (2013): Análisis y perspectivas 2013: desigualdad y derechos sociales, Ministerio de Sanidad, Servicios Sociales e Igualdad, 78 pp.

GOÑI, B. (2008): "Identificación, localización y caracterización de las secciones censales desfavorecidas de la región metropolitana de Barcelona". Scripta Nova. Revista Electrónica de Geografía y Ciencias Sociales. Vol. XII, núm. 272.

HARVEY, D. (1990): La condición de la posmodernidad. Investigación sobre los orígenes del cambio cultural. Buenos Aires, Amorrortu editores.

HERNÁNDEZ AJA, A. (2007): “Áreas vulnerables en el centro de Madrid”. Cuadernos de Investigación Urbanística, $\mathrm{n}^{\circ}$ 53, 5-97. 
HOYT, H. (1939): The Structure and Growth of Residencial neighborhoods in American Cities. Washington D.C, Federal Housing Administration.

INSTITUTO JUAN DE HERRERA (2010): Análisis urbanístico de Barrios Vulnerables en España. Sobre la Vulnerabilidad Urbana, Ministerio de Fomento [http:// www.fomento.gob.es/ NR/rdonlyres/C88DB66D-8669-497C-BEE4-442AE027E2FB/ 111287/SOBRE_vulnerabilidad.pdf]. Fecha de consulta: 16-2-2015.

MALHEIROS, J. (2002) "Ethnic cities: residential patterns in the Northern European and Mediterranean Metropolises. Implications for Policy Design”. International Journal of Population Geography, 2002, nº 8, pp. 107-134

MARTORI, J.C. y HOBERG, K. (2008): "Nuevas técnicas de estadística espacial para la detección de clusters residenciales de población inmigrante". Scripta Nova. Revista Electrónica de Geografía y Ciencias Sociales. Vol. XII, núm. 263.

MASSEY, D.S. y DENTON, N. A. (1988): "The Dimensions of Residential Segregation", Social Forces, Vol. 67 (2), 281-314.

MÉNDEZ, R. Y PRADA-TRIGO, J. (2014): “Crisis, desempleo y vulnerabilidad en Madrid”. Scripta Nova. Revista Electrónica de Geografía y Ciencias Sociales. Vol. XVIII, núm. 474.

MOLLENKOPF, J. y CASTELLS, M. (Eds.) (1991): Dual city: restructuring New York. Nueva York, Russell Sage Foundation.

MORENO, P. Y GARCÍA LÓPEZ, J. (2011): "Estado del arte en procesos de zonificación". GeoFocus, n 11, 155-181

NATERA, J.J. (2012): "Población extranjera en el municipio de Málaga. Evolución de sus niveles de diferenciación residencial y de sus pautas de distribución espacial (2003/2010)". Scripta Nova. Revista Electrónica de Geografía y Ciencias Sociales. Vol. XVI, núm. 413.

OCAÑA OCAÑA, C. (2005): "Microanálisis sociodemográfico de espacios urbanos". Boletín de la Asociación de Geógrafos Españoles, n 40, 5-34

PITARCH, M.D.; ALBERTOS, J.M. y CAÑIZARES, M.C. (2011): “Cambios en la estructura metropolitana durante la última década. El caso del área metropolitana de Valencia", en Gozálvez Pérez, V. y Marco Molina, J. A. (Eds.): Urbanismo expansivo. De la teoría a la realidad. XXII Congreso de Geógrafos Españoles, Universidad de Alicante, pp. 527-539

RUBIALES, M.; BAYONA, J. y PUJADAS, I. (2012): "Patrones espaciales de la segregación residencial en la región metropolitana de Barcelona: Pautas de segregación de los grupos altos". Scripta Nova. Revista Electrónica de Geografía y Ciencias Sociales. Vol. XVI, núm. 423.

SALOM, J. y CASADO, J.M. (2007): "Movilidad cotidiana y mercados de trabajo en la Comunidad Valenciana". Boletín de la Asociación de Geógrafos Españoles, 44, 5-28.

SALOM, J. y PITARCH, M.D. (2014): "El área metropolitana de Valencia en la crisis", en Albertos, J.M. y Sánchez, J.L. (Coords.) Geografía de la crisis económica en España, pp.539-563, PUV-Publicaciones de la Universidad de Valencia, Valencia.

SALOM, J. y ALBERTOS, J.M. (2014): “La crisis económica en los distritos industriales valencianos", en Albertos, J.M. y Sánchez, J.L. (Coords.) Geografía de la crisis económica en España, PUV-Publicaciones de la Universidad de Valencia, Valencia. pp. 467-495. 
SASSEN, S. (1991): The Global City: New York, London, Tokyo. Princeton, Princeton University Press.

SHEVKY, E. Y BELL, W. (1955): Social Area Analysis. Stanford CA. Stanford University Press.

SOJA, E.W. (2008): Postmetrópolis. Estudios críticos sobre las ciudades y las regiones. Madrid. Ed. Traficantes de Sueños.

SORRIBES MONRABAL, J. (1997): Comprendre $i$ gestionar la ciutat: un assaig d'economia i política urbana, Valencia, Universitat de Valencia.

SORRIBES MONRABAL, J. (1999): Las áreas metropolitanas. Análisis teórico y experiencia comparada. Valencia. Consell Metropolitá del'Horta.

TORRES PÉREZ, F. (2007): Nous veïns a la ciutat. Els inmigrants a València i Russafa, PUV, Valencia.

VISAUTA VINACUA, B. (1998): Análisis estadístico con SPSS para Windows, Mc Graw Hill, Madrid. 
